\title{
Sonografie in der Schwangerschaft
}

\section{„Baby-Watching“, Ultraschall nach Mutterschaftsrichtlinien oder Feindiagnostik? DEGUM-Experten klären auf}

Wenn werdende Eltern Ultraschallbilder ihres Ungeborenen in den Händen halten, sind das unvergessliche Momente. Doch vielen reicht das nicht: Sie wollen ihr ungeborenes Kind über große Bildschirme im 3D-Ultraschall sehen. Das sogenannte „Baby-TV“ boomt - doch laut der neuen Strahlenschutzverordnung ist die Sonografie zu solchen kommerziellen Zwecken ab Ende 2020 untersagt. Experten der Deutschen Gesellschaft für Ultraschall in der Medizin e. V. (DEGUM) betonen, dass sonografische Untersuchungen in der Schwangerschaft nur in medizinischen Kontexten angewendet werden sollten. Welche Diagnostik empfehlenswert ist und wo die Unterschiede zwischen dem „Baby-Watching“, feindiagnostischen Untersuchungen und dem Ultraschall nach Mutterschaftsrichtlinien liegen, berichten sie auf der einer Pressekonferenz in Berlin, die Ende April stattgefunden hat.

Das sogenannte „Baby-Watching“ ist eine Ultraschalluntersuchung, die nur dem Betrachten des Fötus dient. Häufig wird dies als eine Art Event mit Familienmitgliedern und Freunden organisiert. Im Prinzip kann jeder so etwas anbieten - im Internet tun dies beispielsweise Hebammen oder Heilpraktiker. „Dabei wird meistens ein 3D- oder ein 4D-Ultraschall eingesetzt, um mit dessen Hilfe das Gesicht, Hände und Füße des Ungeborenen und gegebenenfalls das Geschlecht zu demonstrieren“, sagt PD Dr. Kai-Sven Heling, Vizepräsident der DEGUM. „Mittels 4D-Ultraschall kann auch die Bewegung des Fötus und eventuell seine Mimik gezeigt werden."

Ein medizinischer Hintergrund für das „BabyTV“ bestehe nicht. Heling steht dem Konzept kritisch gegenüber: „Aufgrund von oft wenig qualifizierten Anwendern besteht häufig die Gefahr, dass Fehlbildungen oder sonstige Probleme des Fötus nicht rechtzeitig erkannt werden." Er begrüßt deshalb das zukünftige Verbot solcher kommerziellen Angebote aufgrund der neuen Strahlenschutzverordnung - weist jedoch die dadurch entstandene allgemeine Kritik an Ultraschalluntersuchungen in der Schwangerschaft zurück. „Es gibt keinerlei Erkenntnisse, die einen ultraschallbedingten Schaden am Fötus nachweisen."

Lediglich wenn der sogenannte PW-Dopplerultraschall für einen längeren Zeitraum eingesetzt würde, könnte es zu einem Temperaturanstieg im untersuchten Organsystem kommen. Dies ist jedoch eher theoretisch, da das Dopplersignal im Regelfall nur für einen Zeitraum von wenigen Herzzyklen also ca. für 3 bis 5 Sekunden - aktiviert ist und durch die Blutströmung dieser theoretische Temperatureffekt wieder ausgeglichen wird. Die Dopplersonografie wird im Regelfall in mütterlichen Gefäßen zur Erkennung der Präeklampsie - auch bekannt als Schwangerschaftsvergiftung - und in der Nabelschnur des Fötus eingesetzt. Andere Gefäßgebiete sind ausgesprochen selten und betreffen so gut wie nie einen gesunden Fötus. „Eine potenzielle Gefährdung durch den PW-Dopplerultraschall ist vor diesem Hintergrund also auch von der Hand zu weisen", so Heling.

Im Gegensatz zu kommerziellen Zwecken wie dem „Baby-TV“ - wird die Sonografie zu diagnostischen Zwecken laut der DEGUM hierzulande nur von Ärzten durchgeführt. Laut den Mutterschaftsrichtlinien stehen Schwangeren dafür als Kassenleistung $3 \mathrm{UI}-$ traschalluntersuchungen zur Verfügung 1 in jedem Trimenon. Die erste zwischen der 9. und 12., die zweite zwischen der 19. und 22. und die dritte zwischen der 29. und 32. Schwangerschaftswoche. „Mit den Untersuchungen soll kontrolliert werden, wie sich der Fötus körperlich entwickelt und ob der Arzt auffällige Merkmale entdeckt“, sagt Heling. „So wird auch der Geburtstermin bestimmt und überprüft, ob es sich um eine Mehrlingsschwangerschaft handelt.“

Wenn dabei Auffälligkeiten entdeckt werden oder eine Risikoschwangerschaft vorliegt, übernehmen die Krankenkassen auch die Kosten für feindiagnostische Untersuchungen. Ist das nicht der Fall, müssen Schwangere selbst zahlen, wenn sie die Feindiagnostik wünschen. Damit können mögliche kindliche Fehlbildungen schon in frühen Phasen der Schwangerschaft erkannt werden. „Mit der frühen Ultraschallfeindiagnostik zwischen Anfang der 12. und Ende der 14. Schwangerschaftswoche können wir zahlreiche relevante Fehlbildungen frühzeitig erkennen“, so der DEGUM-Experte.

Bei der feindiagnostischen Untersuchung um die 20. Schwangerschaftswoche könne dann mit sehr hoher Sicherheit festgestellt werden, ob mit der körperlichen Entwicklung des Kindes alles in Ordnung ist. „Hier können das Herz, die Arme und Beine sowie Gehirn und Gesicht des Fötus vollständig untersucht werden“, erläutert Heling.

Da ohne diese Untersuchung potenzielle Fehlbildungen beim Fötus - wie etwa Herzfehler - unentdeckt bleiben, fordert die DEGUM eine Aufnahme dieser Leistungen in den Regelkatalog der Krankenkassen. 